\title{
Total Knee Arthroplasty in Patients With Valgus Deformity
}

\author{
Mahmoud Jabalameli, ${ }^{1}$ Abolfazl Bagherifard, ${ }^{1}$ Hosseinali Hadi, ${ }^{1}$ and Iman Qomashi ${ }^{1,}$ \\ ${ }^{1}$ Bone and Joint Reconstruction Research Center, Shafa Orthopedic Hospital, Iran University of Medical Sciences, Tehran, IR Iran \\ "Corresponding author: Iman Qomashi, Bone and Joint Reconstruction Research Center, Shafa Orthopedic Hospital, Iran University of Medical Sciences, Tehran, IR Iran. Tel: \\ +98-9121987381, E-mail: imanqomashi@yahoo.com
}

Received 2015 November 17; Revised 2016 January 09; Accepted 2016 April 15.

\begin{abstract}
Background: Valgus deformity (more than $10^{\circ}$ valgus on anatomical measurement) is seen in about $10 \%$ of patients undergoing total knee arthroplasty (TKA). Valgus deformity is a challenge for the surgeons both for gap balancing and constraint choice.

Objectives: The aim of this study was to describe a surgical technique to perform TKA in valgus knee.

Patients and Methods: We retrospectively reviewed 32 primary TKA in 27 patients ( 25 females and 2 males), with valgus knee deformity performed since 2006 to 2014 . All procedures have been done by the senior author. In the balancing stage of each procedure, the iliotibial band has been released completely in the first step, and then step by step pie crusting of other lateral structures has been done as needed. Preoperative and postoperative data were obtained retrospectively through chart review and review of radiographs. All patients were clinically examined by the senior author. Preoperative and postoperative knee society scores (KSSs) and functional knee society score (functional KSS) were obtained accordingly.

Results: The patients had an average age of 68 years (range, 52 - 83 years) with an average postoperative follow-up period of 47.5 months (range, 12 - 100 months). The average KSS improved significantly postoperatively from 42 (range, 20 - 69 ) to 90 (range, 67 99) $(\mathrm{P}<0.0001)$. The average functional KSS improved significantly $(\mathrm{P}<0.0001)$ from 51.4 (range, $35-70)$ to 85.5 (range, $45-100)$ during follow-up. An average preoperative range of motion also improved by 15.6 degrees during the last follow-up visit $(\mathrm{P}<0.001)$. We had no complication in our patients.

Conclusions: The results of our study show that complete release of the iliotibial band and step by step pie-crusting of other lateral structures correct valgus deformities in a safe and simple manner with high success rate and less constrained prosthesis.
\end{abstract}

Keywords: Total Knee Arthroplasty, Valgus Deformity, Surgical Technique

\section{Background}

Valgus deformity (more than $10^{\circ}$ valgus on anatomical measurement) is seen in about $10 \%$ of patients undergoing total knee arthroplasty (TKA) (1). This deformity can be congenital or secondary to osteoarthritis, posttraumatic arthritis, rheumatic diseases or an overcorrection in a valgus osteotomy (2).

One of the primary goals of TKA is a stable and wellaligned joint, which is important for satisfactory longterm clinical results and implant survivorship $(3,4)$. This goal is achieved by accurate implant positioning and soft tissue balancing (5-8). Excessive preoperative deformity predispose to higher risk of unsatisfied results in comparison with less deformed knees particularly if the aim is to correct the deformity with soft tissue balancing to use the least amount of constraint (2).

Valgus deformity has two major components, bony structures and soft tissue. Bony changes are lateral cartilage erosion, lateral condylar hypoplasia and metaphysical femur and tibia remodeling. Soft tissue change is primarily from tightening of lateral structures such as iliotibial band (ITB), lateral collateral ligament (LCL), popliteus tendon, posterolateral capsule (PLC), hamstring tendons and the lateral head of the gastrocnemius (LHG) (9).

Three types of valgus deformity have been described. Type1: Less than $10^{\circ}$ mechanical valgus, passively correctable with contraction of lateral structures without elongation of medial collateral ligament (MCL). Type 2: Mechanical valgus between $10^{\circ}$ to $20^{\circ}$ with contracted lateral structures and elongated but functional MCL. Type3: More than $20^{\circ}$ mechanical valgus deformity with tight lateral structures and nonfunctional MCL (2).

For these reasons, valgus deformity is a challenge for the surgeons both for gap balancing and constraint choice.

\section{Objectives}

The aim of this study was to describe a surgical technique to perform TKA in valgus knee and evaluate the results of this surgical technique. 


\section{Patients and Methods}

After ethic committee's approval, we retrospectively reviewed documents of 1070 patients who have had TKA performed by the senior author since 2006 to 2014 .

We included all patients with valgus deformity in the study and called them to visit and exam the knee and the KSS and functional KSS forms were completed for them. We excluded revision knee surgeries.

Finally, 32 primary TKA in 27 patients (5 patients had bilateral surgery) had found. Twenty-five patients were female and 2 were male).

The patients with a preoperative knee valgus angle of $<$ $=10$ degrees were labeled as a group A, while the patients with valgus values larger than 10 degrees were labeled as a group B. (In the other words, patients with type 2 and type 3 valgus deformity labeled as a group B.)

Measurement of preoperative valgus angles was carried on using long standing weight bearing 3-joint X-rays and the preoperative and postoperative knee joint range of motion (ROM) was measured clinically with the senior surgeon.

All surgical procedures were done using a straight skin incision and medial parapatellar approach by the senior author. The distal femur cut was referenced off an intramedullary guide in the femur. Valgus cut was determined on the basis of the angle between femoral mechanical and anatomical axis in the weight bearing 3-joint X-rays. The articular surface of the tibia was resected perpendicular to the anatomic axis using an extramedullary guide. A lateral release was used in $82 \%$ of the cases. It was done when the lateral structures were tight in extension and/or in flexion and the gap configuration was trapezoid. Lateral release started with complete release of ITB in the level of tibia, if lateral tightness remained; first posterior femur cut with rotation on the basis of trans-epicondylar axis was done and then posterior osteophytes were removed. Extension gap was checked again and if the gap is trapezoid pie-crusting of contracted lateral structures (PLC, LCL and popliteus tendon) was done step by step till the rectangular space achieved. Six knees need no lateral release. Nine knees balanced with ITB release alone. Twelve knees need ITB release and PLC pie-crusting. One knee needs PLC and LCL pie-crusting in addition to ITB release. Two knees need PLC and LCL and popliteus tendon pie-crusting in addition to ITB release. Two knees need PLC and popliteus tendon pie-crusting in addition to ITB release. After balancing of soft tissue, in all but four cases nonconstrained PS prosthesis applied. In two cases, the condylar constrained knee (CCK) prosthesis applied because of unbalanced medial/lateral sides (Figure 1) and also in two other cases because of mismatch in flexion/extension gap. Three of this four had type 3 valgus deformities and one had type 2 valgus deformity. Also, in two knees with type 3 deformity PS prosthesis applied. All procedures that need PS prosthesis were done with Nex-Gen prosthesis (Zimmer, USA).

Preoperative and postoperative data were obtained retrospectively through chart review and review of radiographs. All patients were clinically examined by the senior author. Knee society scores (KSS) and functional knee society score (functional KSS) (10) were obtained accordingly.

Statistical analysis of the preoperative and postoperative data was performed using the SPSS Statistics software version 17.0 (SPSS Inc., Chicago, USA). The significance level was defined as $P$ value of less than 0.05 . The Mean values of preoperative and postoperative data were compared using the paired sample t-test for all cases. The mean values of variables were compared using the independent samples t-test between the groups A and B. The variables were correlated using the Pearson's correlation.

\section{Results}

We had 32 TKA in 27 patients. Twenty-five patients were female and 2 were male. The patients had an average age of 68 years (SD: 7.8) with an average postoperative followup period of 47.5 months (range, 12 - 100). The prevalence of valgus deformity was about 3\% of all TKA performed in this time period by the senior author. The preoperative diagnoses were osteoarthritis in 23 patients, rheumatoid arthritis in three patients, and previous high tibia osteotomy (HTO) in one patient. The average preoperative mechanical valgus angle on weight bearing films was $13^{\circ}$ (SD: 8.4) (Table 1).

The average KSS improved significantly postoperatively from 42 (range, 20 - 69) to 90 (range, 67 - 99) (Table 2, $\mathrm{P}<0.0001$ ) with 29 (91\%), 2 (6\%) and 1 (3\%) knees having excellent, good and fair results during the last follow-up visit, respectively.

The average functional KSS improved significantly $(\mathrm{P}<$ 0.0001 ) from 51.4 (range, $35-70$ ) to 85.5 (range, $45-100$ ) during follow-up (Table 2); with 26 (82\%), 4 (12\%), $1(3 \%$ ) and 1(3\%) knees having excellent, good, fair and poor results in the last visit, respectively.

Moreover, an average preoperative ROM improved by 15.6 degrees during the follow-up (Table $2, \mathrm{P}<0.001$ ).

The average preoperative alignment on AP weight bearing radiographs was $13^{\circ}$ valgus (range, $2^{\circ}-34^{\circ}$ ) reaching $4.5^{\circ}$ anatomic valgus angle (range, $0^{\circ}-10^{\circ}$ ) during followup.

The preoperative valgus angle had a moderate inverse correlation with postoperative ROM $(\mathrm{r}=-0.432, \mathrm{P}<0.05)$. The knee society score change had a strong inverse correlation with preoperative KSS $(r=-0.871, P<0.0001)$. None 

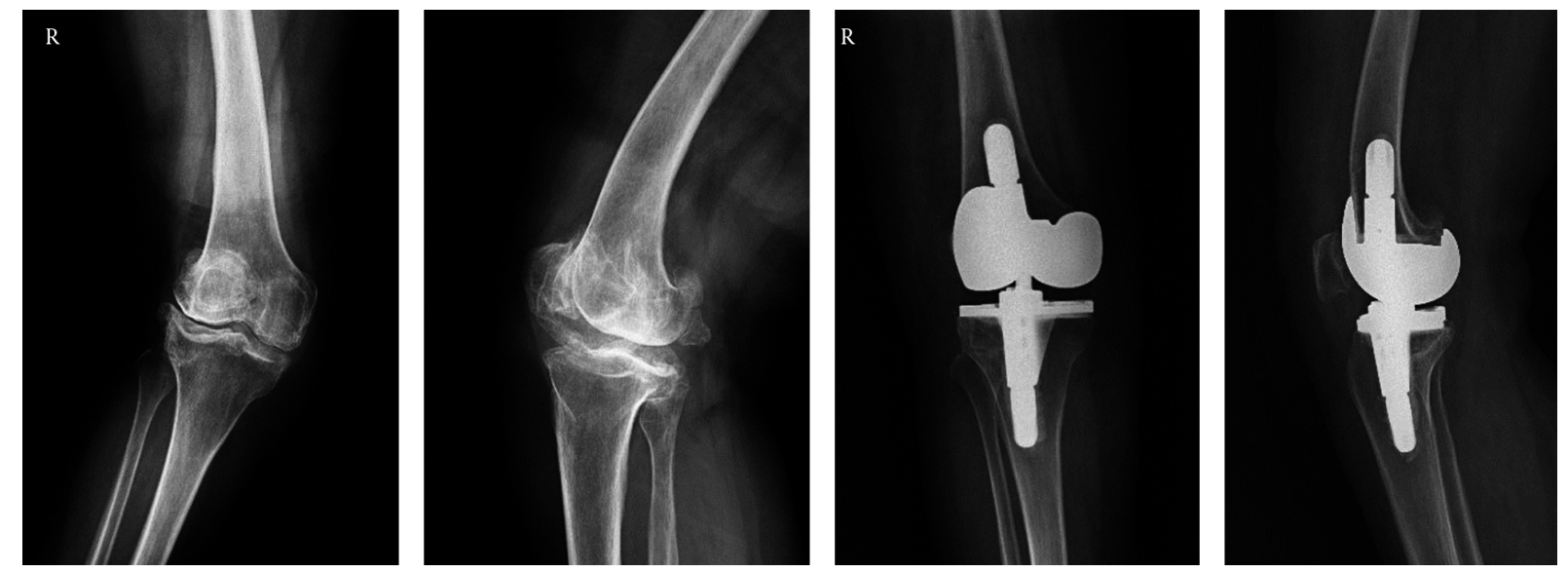

Figure 1. Preoperative and Postoperative X-ray of a Patient With Type 3 Valgus Deformity

Table 1. Baseline Characteristics of the Subjects

\begin{tabular}{|c|c|c|}
\hline & Mean (SD) & Range \\
\hline Age, $y$ & $68.3(7.8)$ & $52-83$ \\
\hline Preoperative valgus angle, degrees & $13(8.4)$ & $2-34$ \\
\hline Preoperative knee range of motion & $100.6(23.5)$ & $45-140$ \\
\hline
\end{tabular}

Table 2. Comparison of Main Variables in All Patients ${ }^{\mathrm{a}}$

\begin{tabular}{|c|c|c|c|c|c|}
\hline & Preoperative & Postoperative & Mean Difference (SD) & 95\% Confidence Interval & P Value \\
\hline Knee society score & 41.3 & 90.1 & $-48.7(15.0)$ & $-54.1--43.3$ & $<0.0001$ \\
\hline Knee society functional score & 51.4 & 85.5 & $-34.1(11.4)$ & $-38.2--30.0$ & $<0.0001$ \\
\hline Knee range of motion, degrees & 100.6 & 116.3 & $-15.6(24.0)$ & $-24.3--7.0$ & 0.001 \\
\hline
\end{tabular}

${ }^{\text {a }}$ Paired sample t-test; the significance level: P value $<0.05$.

of the postoperative changes in ROM, KSS and functional KSS were statistically correlated to the preoperative valgus angle $(\mathrm{P}>0.05)$.

Twelve patients were categorized in the group A, and the rest (20 patients) belonged to the group $B$. While there was a significant difference in the preoperative knee valgus angle (4.4 vs. 18.1, $\mathrm{P}<0.01$ ), there was no statistically significant difference in age, changes in ROM, KSS and functional KSS between the two groups.

\section{Discussion}

Adequate soft tissue balancing in valgus TKA is a challenging issue. Extensive soft tissue release caused major complications such as lateral instability and proneal nerve palsy. The purpose of this study is to verify whether a com- plete release of the iliotibial band and step by step piecrusting of lateral structures can correct the valgus deformity and restore the knee function.

The prevalence valgus knee in our study was about 3\% that was lower than other studies (1). However, a study by Tahmasebi et al. (11) showed the same result. Our lower result may be because of missed patients in this long-time interval or may be because of different population specifies according to similar result in a study in our country (11).

In our study, 29 (91\%) and 26 (82\%) knees had excellent KSS and functional KSS, respectively in the last visit (a midterm result), which is comparable with short and mid-term results of other studies with selective lateral release techniques (12-14). All these techniques were common in these properties: 1) midsubstance release of ligaments in extension; 2) inside-out release; 3) not all lateral structures re- 
leased, and 4) popliteus tendon was remained intact. In our technique (pie-crusting technique), the distinguishing property is gradual release of tight lateral structures in extension under maximum tension with the use of spacer blocks without complete release of LCL or PLC.

We had no proneal nerve palsy. The risk of proneal nerve injury is possible when pie-crusting of posterolateral corner of knee in the level of joint line has done. An MRI study demonstrates 9 - $22 \mathrm{~mm}$ distance of proneal nerve from the posterolateral corner, which is separate from the bone with lateral head of the gastrocnemius (15).

Clarke et al. (16) and Elkus et al. (17) studies with 2 and 5 years follow-up respectively had excellent and good results with no residual instability. In our study, there is no residual instability too. The iliotibial band and the posterolateral capsule are varus restraints mainly in extension and are less effective in flexion. The lateral collateral ligament has a major varus stabilizing effect in extension but acts as a varus stabilizer in flexion as well. Popliteus tendon mainly is a flexion stabilizer with minimal effects in extension. When the cumulative effect of sequential releases is investigated, when the popliteus tendon is excised, significant varus instability occurs in flexion. In our study, the popliteus tendon was released only in two knees and left intact in all other knees. So, we had no instability in our last follow-up visits.

All but four cases (which had severe valgus deformity more than 25 degree) finished with nonconstrained prosthesis and this is an advantage for this method to do with less constrain.

Clinical findings (ROM, KSS and functional KSS) between the group A and B had no significant differences, in other words there was no differences in results according to degree of valgus deformity; so, this is an appropriate technique in all types of valgus deformities.

The results of the current study show that complete release of the iliotibial band and step by step release of other lateral structures by pie-crusting can correct valgus deformities in a safe and simple manner with high success rate and less constrained prosthesis and without serious complications. Thus, this method can be an appropriate method for TKA in valgus knees.

\section{Footnote}

Authors' Contribution: Study design: Mahmoud Jabaameli; data acquisition: Abolfazl Bagherifard, Iman Qomashi; manuscript preparation and revision: Hoseinali Hadi, Iman Qomashi, Abolfazl Bagherifard; study supervision: Mahmoud Jabalameli.

\section{References}

1. Ranawat AS, Ranawat CS, Elkus M, Rasquinha VJ, Rossi R, Babhulkar S. Total knee arthroplasty for severe valgus deformity. J Bone Joint Surg Am. 2005;87 Suppl 1(Pt 2):271-84. doi: 10.2106/JBJS.E.00308. [PubMed: 16140800].

2. Rossi R, Rosso F, Cottino U, Dettoni F, Bonasia DE, Bruzzone M. Total knee arthroplasty in the valgus knee. Int Orthop. 2014;38(2):273-83. doi: 10.1007/s00264-013-2227-4. [PubMed: 24366186].

3. Kurtz S, Ong K, Lau E, Mowat F, Halpern M. Projections of primary and revision hip and knee arthroplasty in the United States from 2005 to 2030. J Bone Joint Surg Am. 2007;89(4):780-5. doi: 10.2106/JBJS.F.00222. [PubMed: 17403800].

4. Matsumoto T, Muratsu H, Kubo S, Matsushita T, Kurosaka M, Kuroda R. The influence of preoperative deformity on intraoperative soft tissue balance in posterior-stabilized total knee arthroplasty. J Arthroplasty. 2011;26(8):1291-8. doi: 10.1016/j.arth.2011.01.003. [PubMed: 21353452].

5. Cameron HU, Hunter GA. Failure in total knee arthroplasty: mechanisms, revisions, and results. Clin Orthop Relat Res. 1982(170):141-6. [PubMed: 7127939].

6. Mihalko WM, Miller C, Krackow KA. Total knee arthroplasty ligament balancing and gap kinematics with posterior cruciate ligament retention and sacrifice. Am J Orthop (Belle Mead NJ). 2000;29(8):610-6. [PubMed: 10955465].

7. Ritter MA, Davis KE, Meding JB, Pierson JL, Berend ME, Malinzak RA. The effect of alignment and BMI on failure of total knee replacement. J Bone Joint Surg Am. 2011;93(17):1588-96. doi: 10.2106/JBJS.J.00772. [PubMed: 21915573].

8. Ritter MA, Davis KE, Davis P, Farris A, Malinzak RA, Berend ME, et al. Preoperative malalignment increases risk of failure after total knee arthroplasty. J Bone Joint Surg Am. 2013;95(2):126-31. doi: 10.2106/JBJS.K.00607. [PubMed: 23324959].

9. Ranawat CS. Total-condylar knee arthroplasty: technique, results, and complications. New York: Springer; 1985.

10. Insall JN, Dorr LD, Scott RD, Scott WN. Rationale of the Knee Society clinical rating system. Clin Orthop Relat Res. 1989(248):13-4. [PubMed: 2805470].

11. Tahmasebi MT, Mortazavi MJ, Haghpanah B. Results of TKA in valgus knees. Oral presentation in Iranian Orthopedic Association congress. 2015; .

12. Insall JN, Easley ME. Surgical techniques and instrumentation in total knee arthroplasty. In: Insall JN, Scott WN, editors. Surgery of the Knee. 3 ed. New York: Churchill Livingstone; 2001. p. 1717-38.

13. Politi J, Scott R. Balancing severe valgus deformity in total knee arthroplasty using a lateral cruciform retinacular release. J Arthroplasty. 2004;19(5):553-7. [PubMed: 15284974].

14. Scott RD. Total Knee Arthroplasty in Severe Valgus Deformity. Philadelphia: WB Saunders Co; 2006. p. 45-53.

15. Clarke HD, Schwartz JB, Math KR, Scuderi GR. Anatomic risk of peroneal nerve injury with the "pie crust" technique for valgus release in total knee arthroplasty. J Arthroplasty. 2004;19(1):40-4. [PubMed: 14716649].

16. Clarke HD, Fuchs R, Scuderi GR, Scott WN, Insall JN. Clinical results in valgus total knee arthroplasty with the "pie crust" technique of lateral soft tissue releases. J Arthroplasty. 2005;20(8):1010-4. doi: 10.1016/j.arth.2005.03.036. [PubMed: 16376256].

17. Elkus M, Ranawat CS, Rasquinha VJ, Babhulkar S, Rossi R, Ranawat AS Total knee arthroplasty for severe valgus deformity. Five to fourteenyear follow-up. J Bone Joint Surg Am. 2004;86-A(12):2671-6. [PubMed: 15590852]. 